\title{
Overexpression of FOXO4 induces apoptosis of clear-cell renal carcinoma cells through downregulation of Bim
}

\author{
WEI WANG $^{1 *}$, PANG-HU ZHOU ${ }^{1,2^{*}}$ and WEI HU ${ }^{1}$ \\ Departments of ${ }^{1}$ Urology and ${ }^{2}$ Orthopedics, Renmin Hospital of Wuhan University, Wuhan, Hubei 430060, P.R. China
}

Received January 18, 2015; Accepted October 22, 2015

DOI: $10.3892 / \mathrm{mmr} .2016 .4789$

\begin{abstract}
Forkhead box O4 (FOXO4) has been reported to be a novel tumor suppressor gene in gastrointestinal cancers; however, its role in clear-cell renal carcinoma cells (ccRCC) has remained largely elusive. The present study assessed the expression levels of FOXO4 in RCC tissues and cells. Furthermore, the effects of vector-mediated overexpression of FOXO4 on the apoptotic rate of the 786-0 and Caki-1 cell lines and the role of Bim in this process were investigated. The results demonstrated that the protein and mRNA expression levels of FOXO4 were decreased in renal cancer tissues and cell lines compared with those in normal tissues and cell lines. FOXO4 overexpression significantly increased the apoptotic rate of ccRCC cells in vitro, along with increased protein expression levels of Bim, cleaved-caspase 3, B-cell lymphoma 2 ( $\mathrm{Bcl}-2$ )-associated $\mathrm{X}$ protein (Bax) and cytochrome $c$, as well as a decrease in $\mathrm{Bcl}-2$ expression. Of note, the apoptotic effects of FOXO4 overexpression in 786-0 cells were inhibited by small interfering RNA-mediated knockdown of Bim. The results of the present study indicated that FOXO4 has an inhibitory effect in ccRCC, at least in part through inducing apoptosis via upregulation of Bim in the mitochondria-dependent pathway.
\end{abstract}

\section{Introduction}

Renal cell carcinoma ( $\mathrm{RCC}$ ) is a common type of malignant neoplasm of the urinary system with increasing morbidity and mortality worldwide (1). Histopathologically, the clear-cell (cc) sub-type accounts for $80 \%$ of all renal-cell carcinoma (2). While surgical approaches represent a curative treatment method for RCC, one-third of patients develop metastasis and

Correspondence to: Dr Pang-Hu Zhou, Department of Orthopedics, Renmin Hospital of Wuhan University, 99 Ziyang Road, Wuhan, Hubei 430060, P.R. China

E-mail: zhoupanghu@gmail.com

${ }^{*}$ Contributed equally

Key words: forkhead box O4, apoptosis, Bim, clear-cell renal carcinoma require systemic therapy (3). However, as end-stage RCC is frequently resistant to radiotherapy and chemotherapy, the affected patients have a poor prognosis. Therefore, novel molecular targets in RCC are required to be identified in order to provide more efficient therapeutic approaches.

Forkhead box transcription factors, class O (FOXO) include four important components: FOXO1, -3, -4 and -6, which have been reported not only to have crucial roles in cellular proliferation, inflammation, stress resistance and apoptosis (4-7), but to also exert anti-tumor effects in certain types of malignant neoplasm $(8,9)$. FOXO4 is highly expressed in kidney, muscle and colorectal tissues, and has protective effects against cardiovascular diseases (10) and malignancies. Studies have reported that FOXO4 exerts tumor-suppressive effects in cholangiocarcinoma (11) and gastric cancer (12), while its potential effect on RCC has remained elusive and was therefore assessed in the present study. For this, the expression of FOXO4 in RCC tissues and cell lines as well as normal renal cells was assessed, and the effects of vector-mediated overexpression of FOXO on the apoptosis of RCC cell lines were investigated. Furthermore, the role of apoptotic proteins in this process was assessed to determine the underlying molecular mechanism.

\section{Materials and methods}

Patients and samples. Fifty-eight human RCC tissue samples and adjacent normal tissues were obtained from RCC patients (49 males and 9 females; age, 46-70 years with mean of 58.6 years) undergoing nephrectomy at the Department of Urology, Renmin Hospital of Wuhan University (Wuhan, China). All of the tumor samples were of the clear-cell sub-type as verified by pathological examination, and the corresponding normal samples obtained from adjacent tissues did not exhibit any pathological abnormalities. The samples were frozen in liquid nitrogen and stored at $-70^{\circ} \mathrm{C}$ prior to protein and RNA extraction. The use of human tissues was approved by the Institutional Review Board of Wuhan University (Wuhan, China) and conformed to the Declaration of Helsinki. All of the patients provided written informed consent.

Cell culture. The 786-0 and HK-2 cell lines were obtained from the American Type Culture Collection (Manassas, VA, USA). The Caki-1 and Caki-2 cell lines were purchased from the China Center for Type Culture Collection (Wuhan, China). 
All cell lines were maintained in RPMI-1640 (Gibco; Thermo Fisher Scientific, Inc., Waltham, MA, USA) supplemented with $10 \%$ fetal bovine serum (FBS; Gibco) and $1 \%(\mathrm{v} / \mathrm{v})$ penicillin/streptomycin (Gibco; Thermo Fisher Scientific, Inc.) at $37^{\circ} \mathrm{C}$ in a humidified atmosphere containing $5 \% \mathrm{CO}_{2}$.

Plasmid construction and purification of cultured 786-0 cells. FOXO4 overexpression plasmids were constructed with pcDNA3.1/Flag (Clontech Laboratories, Inc., Mountainview, CA, USA) as the vector, FOXO4 as the expression gene and flag as a tag. The primer sequences for FOXO4 were as follows: Sense, 5'-ATGGATCCGGGAATGAGAAT-3' and anti-sense, 5'-TCAGGGATCTTGGCTCAAAG-3'. The constructed vector was verified using standard DNA sequencing using an ABI PRISM 310 Genetic Analyzer (PerkinElmer, Inc., Waltham, MA, USA). An empty expression plasmid was used as a control. A total of $1 \times 10^{5}$ cells were seeded into each well of a six-well plate. Upon $70 \%$ confluency, the medium was discarded and replaced with fresh non-supplemented, serum-free medium. A total of $1.5 \mu \mathrm{g}$ pcDNA3.1/Flag/FOXO4 plasmid or pcDNA3.1/Flag was mixed with $100 \mu$ l Opti-MEM (Invitrogen; Thermo Fisher Scientific, Inc.). Furthermore, $4.5 \mu 1$ Lipofectamine 2000 (Invitrogen; Thermo Fisher Scientific, Inc.) was mixed with $100 \mu \mathrm{l}$ Opti-MEM and incubated for $5 \mathrm{~min}$ at room temperature. Subsequently, the plasmid and lipofectamine 2000 solutions were mixed and incubated for another $20 \mathrm{~min}$ prior to addition to the cells. Following $24 \mathrm{~h}$ of incubation, the transfection efficiency was determined by western blot analysis and reverse-transcription quantitative polymerase chain reaction (RT-qPCR) analyses.

Bim small interfering (si)RNA transfection. Bim siRNA and scrambled control siRNA were purchased from Santa Cruz Biotechnology, Inc. (Dallas, TX, USA). Cells were transfected with siRNA following the manufacturer's instructions. In brief, $2 \times 10^{5} 786-0$ cells were cultured in $2 \mathrm{ml}$ antibiotic-free medium in a six-well plate and transfected with pcDNA3.1/Flag/FOXO4 $24 \mathrm{~h}$ prior to treatment with Bim siRNA. First, 2-8 $\mu \mathrm{l}$ of siRNA duplex was added to $100 \mu \mathrm{l}$ siRNA transfection medium (solution A) and 2-8 $\mu \mathrm{l}$ of siRNA transfection reagent was added to $100 \mu 1$ siRNA transfection medium (solution B). Subsequently, solutions A and B were mixed and incubated at room temperate for $30 \mathrm{~min}$. Following washing of each well with $2 \mathrm{ml}$ siRNA transfection medium, $0.8 \mathrm{ml}$ siRNA transfection medium was added to each well containing the siRNA transfection reagent mixture (200 $\mu \mathrm{l}$ from solutions A and B). After incubation for $8 \mathrm{~h}$, the supernatant was removed and replaced with normal medium, followed by incubation for another $24 \mathrm{~h}$ prior to analysis. The silencing efficiency was examined using western blot and RT-qPCR. All assays, including transfections, western blots and PCR, were performed as at least three independent experiments.

Flow-cytometric cell sorting (FACS) analysis of cell apoptosis. ccRCC cells were transfected with pcDNA3.1/Flag/FOXO4 or pcDNA3.1/Flag and incubated for $24 \mathrm{~h}$. Subsequently, all cultured cells and the media were collected, and following centrifugation at $1,000 \mathrm{x}$ g for $5 \mathrm{~min}$, cells were re-suspended in $300 \mu \mathrm{l}$ ice-cold binding buffer. Cells were then stained with $10 \mu \mathrm{l}$ propidium iodide (PI) and $5 \mu \mathrm{l}$ Annexin V-fluorescein isothiocyanate (FITC) using an Annexin V-FITC kit (Beyotime Institute of Biotechnology, Haimen, China) and incubated for another $5 \mathrm{~min}$. Ten thousand events were analyzed to determine the proportion of apoptotic cells using a FACSCalibur flow cytometer (Becton Dickinson, Franklin Lakes, NJ, USA).

Western blot analysis. Tissue and cell extracts were obtained using radioimmunoprecipitation assay lysis buffer (Biyuntian Biotechnology Co., Shanghai, China) on ice and the lysates were centrifuged for $15 \mathrm{~min}\left(12,000 \mathrm{x} \mathrm{g}, 4^{\circ} \mathrm{C}\right)$. Protein concentration was determined using the QuantiPro ${ }^{\mathrm{TM}}$ BCA assay kit (Sigma-Aldrich, St. Louis, MO, USA). Proteins $(1 \mu \mathrm{g} / \mu \mathrm{l} ; 20 \mu \mathrm{l})$ were separated by $10 \%$ sodium dodecyl sulfate polyacrylamide gel electrophoresis (Shanghai Haling Biotechnology Co., Ltd., Shanghai, China) and subsequently electroblotted onto polyvinylidene fluoride membranes (Millipore, Billerica, MA, USA). After blocking non-specific binding to the membrane with $5 \%$ non-fat milk, the membranes were incubated overnight at $4^{\circ} \mathrm{C}$ with the following primary antibodies at a 1:1,000 dilution: Rabbit monoclonal anti-FOXO4 (Abcam, Cambridge, MA, USA; cat. no. ab128908), rabbit polyclonal anti-Bim (Abcam; cat. no. ab7888), rabbit polyclonal anti-B-cell lymphoma 2 (Bcl-2; Abcam; cat. no. ab59348), rabbit monoclonal anti-Bcl-2-associated X (Bax; Abcam; cat. no. ab32503), rabbit monoclonal anti-cleaved-caspase 3 (Cell Signaling Technology, Inc., Danvers, MA, USA; cat. no. 9664), rabbit polyclonal anti-cytochrome $c$ (Abcam; cat. no. ab90529) and rabbit polyclonal anti- $\beta$-actin (Santa Cruz Biotechnology, Inc.; cat. no. sc-130657). Membranes were washed three times with Tris-buffered saline with Tween 20 (TBST; Wuhan Guge Biotechnology Co., Ltd, Wuhan, China) and incubated with corresponding IRDye horseradish peroxidase-conjugated goat anti-rabbit secondary antibody (LI-COR Biosciences, Lincoln, NE, USA; cat. no. 925-32211) for $2 \mathrm{~h}$ at room temperature. The membranes were rinsed three times with TBST and the protein bands were visualized using a two-color infrared imaging system (Odyssey; LI-COR Biosciences).

$R T-q P C R$. Total RNA was isolated from the kidney tissues and cultured cells with TRIzol reagent (Invitrogen; Thermo Fisher Scientific, Inc.). cDNA was synthesized using the PrimeScript $^{\text {TM }}$ RT reagent kit (Takara Bio, Inc., Otsu, Japan) according to the manufacturer's instructions. RNA $(1.5 \mu \mathrm{g})$ extracted from tissues was used as a template to perform one-step RT-PCR, and human glyceraldehyde 3-phosphate dehydrogenase (GAPDH) was used as an internal control. The resulting cDNA was amplified using real-time PCR in a volume of $20 \mu \mathrm{l}$ with the SYBR Green Master mix (Takara Bio, Inc.) method and the ABI 7500 Real-Time RT-PCR system (Thermo Fisher Scientific, Inc.). The conditions of the PCR were initial denaturation at $95^{\circ} \mathrm{C}$ for $30 \mathrm{sec}$, followed by 40 cycles of $5 \mathrm{sec}$ at $95^{\circ} \mathrm{C}, 30 \mathrm{sec}$ at $60^{\circ} \mathrm{C}$ and $1 \mathrm{~min}$ at $72^{\circ} \mathrm{C}$. The $\mathrm{Cq}$ values of each samples were calculated using the $2^{-\Delta \Delta \mathrm{Cq}}$ data analysis method (13). The primer sequences were generated by Sangon Biotech Co., Ltd. (Shanghai, China), as follows: GAPDH forward, 5'-AGAAGGCTGGGG CTCATTTG-3' and reverse, 5'-AGGGGCCATCCACAG TCTTC-3'; and FOXO4 forward, 5'-CTTTCTGAAGACTGG CAGGAATGTG-3' and reverse, 5'-GATCTAGGTCTATGA TCGCGGCAG-3'. 


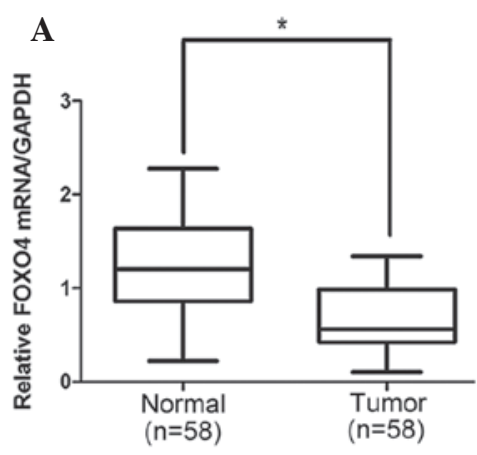

D

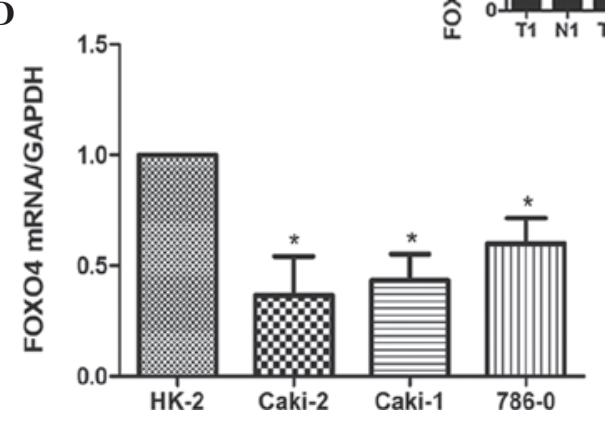

B

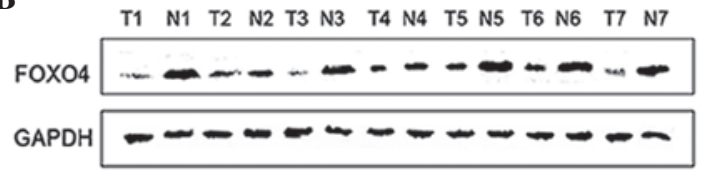

C

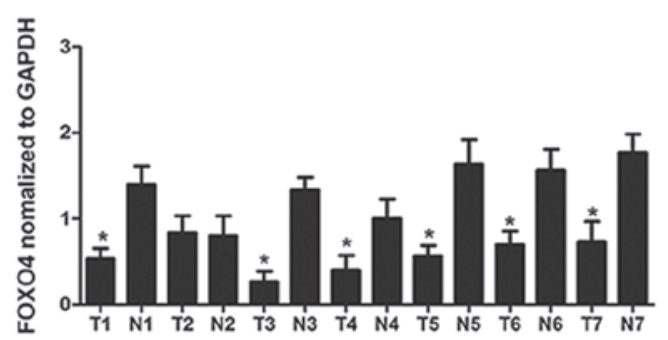

T8 N8 T9 N9 T10 N10 T11 N11 T12 N12 T13 N13
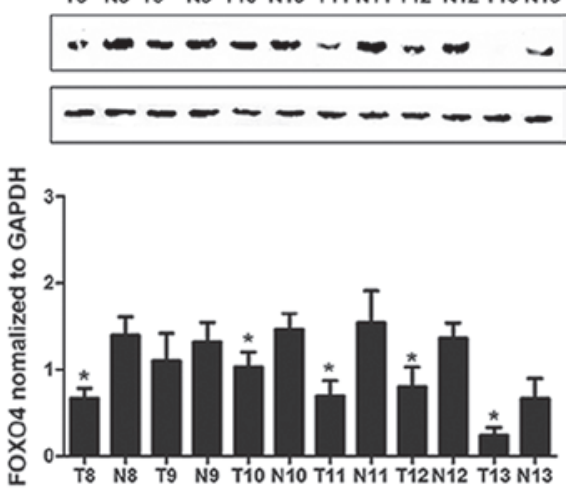

$\mathbf{E}$

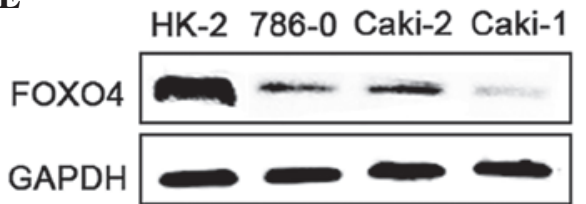

Figure 1. FOXO4 is downregulated in RCC tissues and cell lines. (A) FOXO4 mRNA expression in clinical RCC specimens (T) and normal kidney tissues (N). Boxes indicate the upper and lower bounds and the bars indicate the SEM. (B) Western blot analysis of FOXO4 protein expression in thirteen RCC tissues (T) and adjacent normal kidney tissues (N). (C) Statistical analysis of FOXO4 protein expression between RCC and normal tissues. (D) Quantified FOXO4 mRNA expression in the HK2 normal kidney cell line and ccRCC cell lines. (E) Western blot analysis of FOXO4 protein expression in HK2 and ccRCC cell lines. Values are expressed as the mean \pm SEM from three separate experiments using the same sample * $\mathrm{P}<0.05$, T vs. N or RCC cell lines vs. HK-2. FOXO4, forkhead box O4; T, RCC tissues; N, normal kidney tissues; ccRCC, clear-cell renal carcinoma cells, SEM, standard error of the mean.

A

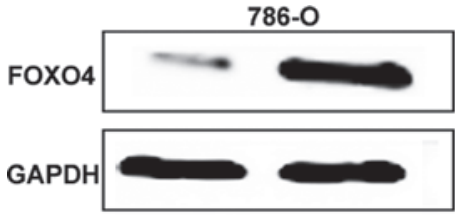

C

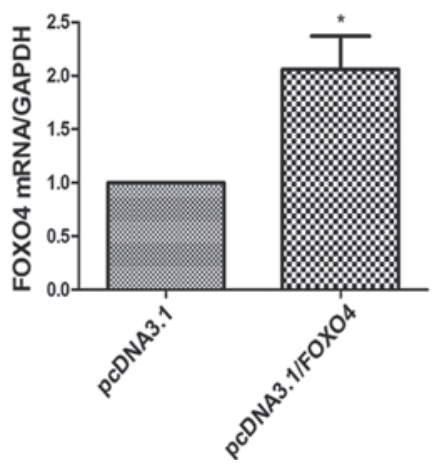

B

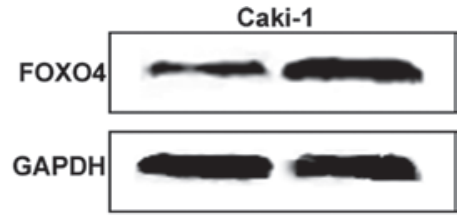

D

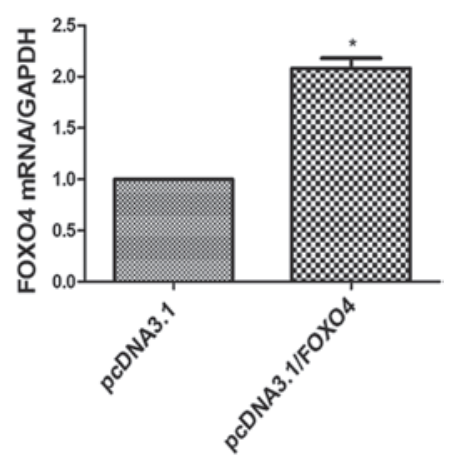

Figure 2. Enhanced expression of FOXO4 in 786-0 and Caki-1 cells. (A and B) Representative western blots of the expression of FOXO4 in 786-0 and Caki-1 cells transfected with pcDNA3.1 or pcDNA3.1/FOXO4. (C and D) Relative mRNA levels of FOXO4 after transfection detected by reverse-transcription quantitative polymerase chain reaction analysis. Values are expressed as the mean \pm standard error of the mean of experiments performed in triplicate. "P $<0.05$, pcDNA3.1 group vs. pcDNA3.1/FOXO4 group. pcDNA3.1/FOXO4, FOXO4 overexpression vector; pcDNA3.1, empty vector; FOXO4, forkhead box O4.

Statistical analysis. Experiments were repeated three times independently and representative data are shown. Values are expressed as the mean \pm standard error of the mean. Student's $t$-test was used for comparison between two groups, and one-way analysis of variance was used for comparisons between groups. $\mathrm{P}<0.05$ was considered to indicate a statistically significant difference between values.

\section{Results}

FOXO4 is downregulated in RCC tissues and cell lines. In order to examine expression levels of FOXO4, 58 clinical renal cancer samples were obtained and analyzed using RT-qPCR. As shown in Fig. 1A, FOXO4 expression was significantly reduced in 51 ( $87.9 \%$ of total) human RCC tissues compared 
A
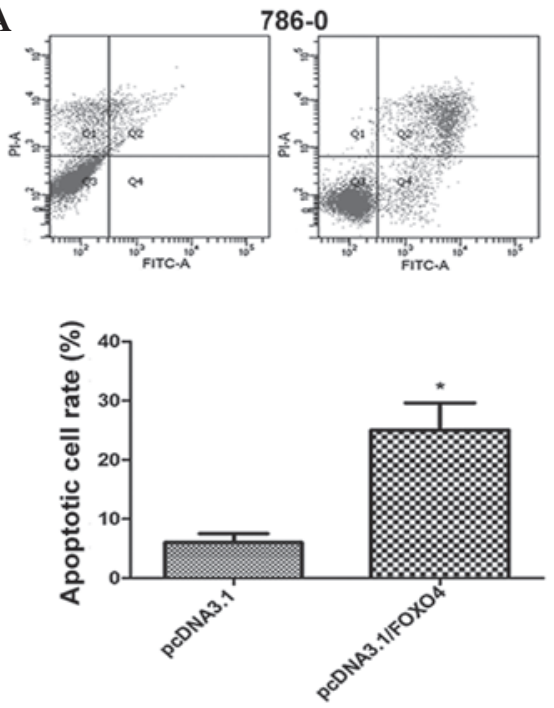

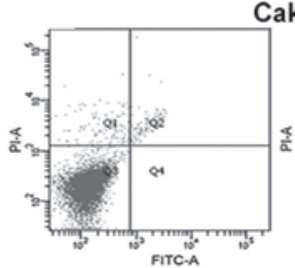

Caki-1
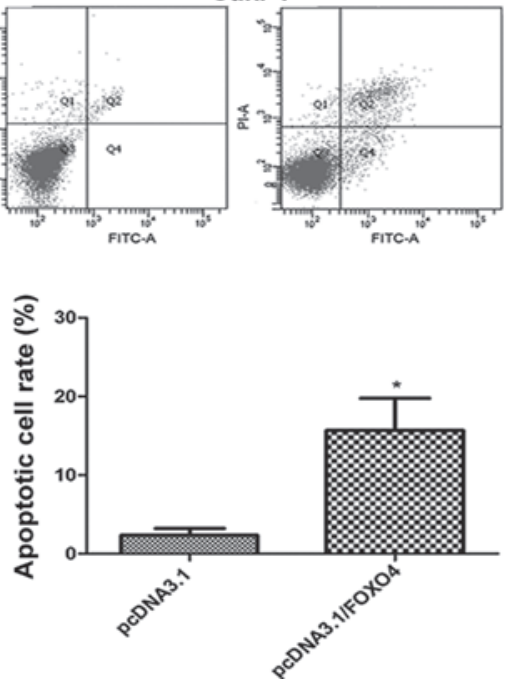

B
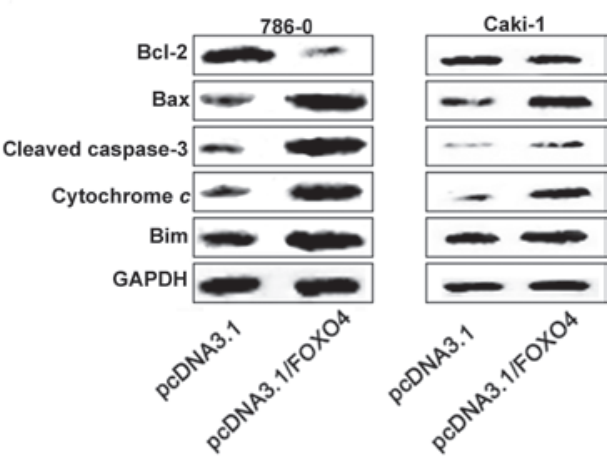

Figure 3. Overexpression of FOXO4 caused significant apoptosis in clear-cell renal carcinoma cells. (A) Apoptotic rate of 786-0 and Caki-1 cells measured by Annexin V/PI double staining. Results are representative of at least three repeats. Values are expressed as the mean \pm standard error of the mean of experiments performed in triplicate. "P<0.05, pcDNA3.1 group vs. pcDNA3.1/FOXO4 group. (B) Representative western blots of apoptosis-associated proteins in 786-0 and Caki-1 after transfection. pcDNA3.1/FOXO4, FOXO4 overexpression vector; pcDNA3.1, empty vector; FOXO4, forkhead box O4; PI, propidium iodide; FITC, fluorescein isothiocyanate; Bcl-2, B-cell lymphoma 2; Bax, Bcl-2-associated X protein.

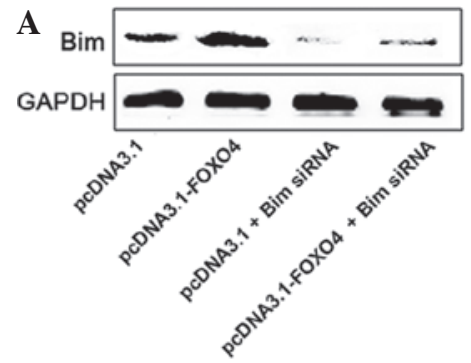

C

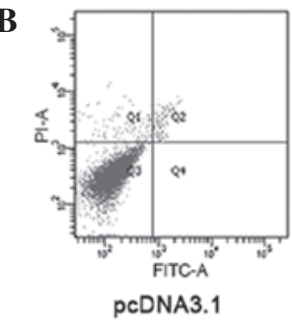

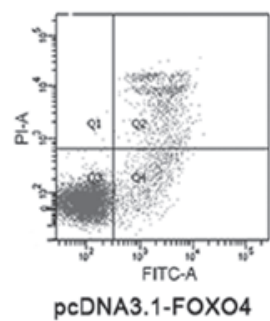

PCDNA3.1-FOXO4
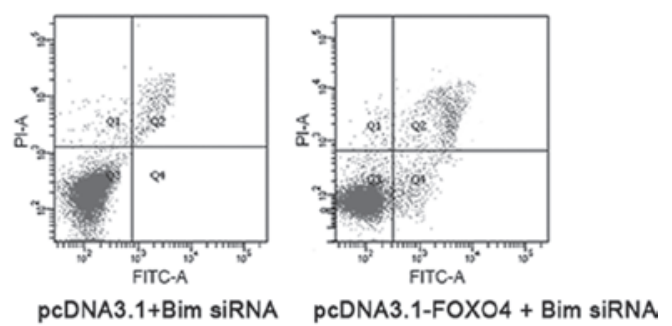

D
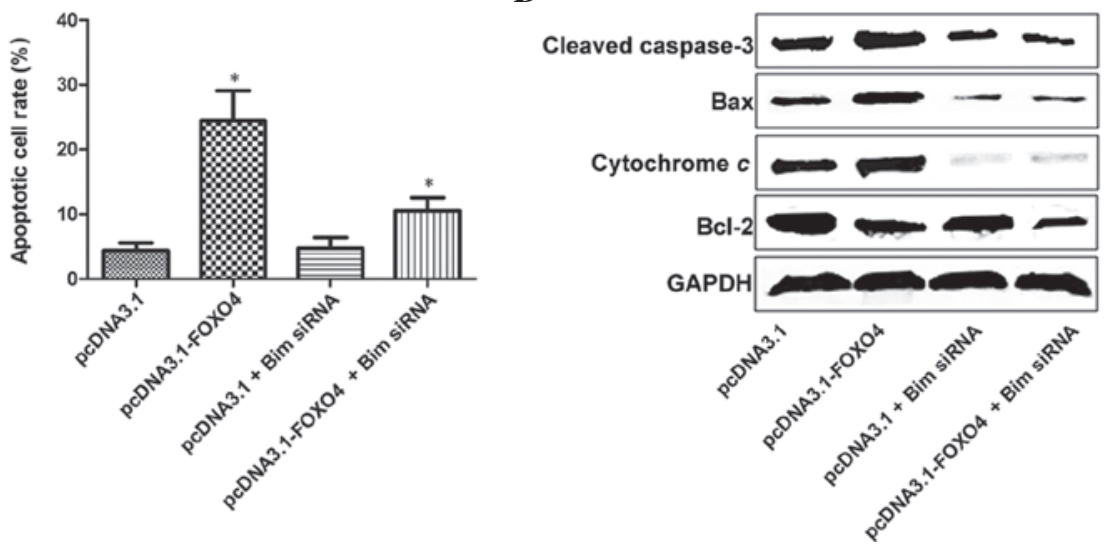

Figure 4. Silencing of Bim partly rescued 786-0 cells from apoptosis induced by FOXO4 overexpression. (A) Representative wester blot of FOXO4 protein expression demonstrating a marked suppression of FOXO4 following Bim knockdown. (B and C) Knockdown of Bim expression in FOXO4-overexpressing 786-0 cells attenuated the apoptotic rate compared to that in control plasmid-transfected 786-0 cells. Values are expressed as the mean \pm standard error of the mean of experiments performed in triplicate ${ }^{*} \mathrm{P}<0.05$, pcDNA3.1 group vs. pcDNA3.1-FOXO4 group. (D) Representative western blots of apoptosis-associated proteins in 786-0 cells with Bim knockdown/FOXO4 overexpression. pcDNA3.1/FOXO4, FOXO4 overexpression vector; pcDNA3.1, empty vector; FOXO4, forkhead box O4; PI, propidium iodide; FITC, fluorescein isothiocyanate; Bcl-2, B-cell lymphoma 2; Bax, Bcl-2-associated X protein; siRNA, small interfering RNA.

with that in their corresponding adjacent non-tumorous tissues. Furthermore, the FOXO4 protein expression was assessed in 13 pairs of randomly selected RCC tissues and their adjacent normal tissues using western blot analysis. As shown in Fig. 1B and C, the protein levels of FOXO4 were significantly decreased in RCC tissues compared with those in their corresponding normal tissues in 11 out of 13 pairs $(\mathrm{P}<0.05)$. In addition, as shown in Fig. 1D and $\mathrm{E}$, the relative expression levels of FOXO4 mRNA and protein in the three RCC cell lines 786-0, Caki-1 and Caki-2 were significantly 
reduced compared to those in the HK2 normal human renal tubular cell line.

Plasmid-mediated FOXO4 overexpression in ccRCC cell lines. In order to clarify the role of FOXO4 in ccRCC cells, two ccRCC cell lines were transfected with pcDNA3.1/FOXO4, which resulted in high expression of FOXO4. As shown in Fig. 2, western blot and RT-qPCR indicated that in 786-0 and Caki-1 cells, FOXO4 protein and mRNA expression in the pcDNA3.1/FOXO4 plasmid group was upregulated compared with that in the empty plasmid group.

FOXO4 overexpression induces apoptosis in ccRCC cells. Following transfection with pcDNA3.1/FOXO4 plasmid or pcDNA3.1 plasmid for $24 \mathrm{~h}$, the apoptotic rate was assessed by FACS using Annexin V/PI double staining. The results revealed that overexpression of $\mathrm{FOXO} 4$ significantly increased the apoptotic rate of 786-0 $(25.0 \pm 8.0)$ and Caki-1 $(15.9 \pm 4.1)$ cells, while the apoptotic rate of the control-transfected cells was $3.1 \pm 0.2$ in $786-0$ cells and $1.3 \pm 0.2$ in Caki- 1 cells (Fig. 3A). To identify the mechanism of FOXO4-induced apoptosis in 786-0 cells, FOXO4-overexpressing cells were subjected to western blot analysis of apoptosis-associated proteins at $24 \mathrm{~h}$ following transfection. As shown in Fig. 3B, Bax, cleaved-caspase 3, cytochrome $c$ and Bim were upregulated and Bcl-2 was downregulated in 786-0 and Caki-1 cells transfected with pcDNA3.1/FOXO4.

FOXO4 overexpression induces apoptosis of 786-0 cells partly through upregulation of Bim. In order to assess the role of Bim in FOXO4-induced apoptosis, Bim siRNA was used to suppress the expression of Bim in FOXO4-transfected 786-0 cells or control-transfected cells. As shown in Fig. 4A, western blot analysis indicated that Bim was downregulated after siRNA treatment. Of note, knockdown of Bim expression led to a substantial decrease in the apoptotic rate of FOXO4-overexpressing 786-0 cells $(\mathrm{P}<0.05)$ (Fig. 4B and $\mathrm{C})$. In addition, the expression of apoptosis-associated proteins was altered following knockdown of Bim. In the pcDNA3.1-FOXO4 + Bim-siRNA group, cleaved-caspase 3, Bax and cytochrome $c$ were significantly downregulated compared with those in the pcDNA3.1-FOXO4 group. However, knockdown of Bim was not able to rescue the levels of Bcl-2, which were significantly downregulated in the pcDNA3.1-FOXO4 group (Fig. 4D).

\section{Discussion}

The present study was the first to demonstrate the tumor-suppressor role of FOXO4 in ccRCC. FOXO4 was shown to be downregulated in renal cancer tissues but to be highly expressed in non-tumorous tissues. In accordance with the expression levels in tissues, FOXO4 was also expressed at high levels in the HK-2 normal human renal proximal tubular cell line, while its expression in several renal cancer cell lines was low. These results suggested that FOXO4 may act as a negative regulator in RCC. Of note, vector-mediated overexpression of FOXO4 caused a significant level of apoptosis in 786-0 and Caki-1 cells in vitro, alongside decreased levels of Bax, cleaved-caspase 3, cytochrome $c$ and Bim, and increased expression of Bcl-2. Furthermore, knockdown of Bim attenuated the apoptotic effects of FOXO4, indicating that FOXO4-induced apoptosis is, at least partially, mediated via Bim.

A number of studies have shown that phosphorylation of FOXO proteins by Akt promote apoptosis in cancer cells (14-16). Roy et al (14) reported that the activation of FOXO transcription factor led to cell cycle arrest and apoptosis in pancreatic cancer, suggesting its tumor-suppressive effects. Furthermore, Nakayoshi et al (17) found that FOXO4 knockdown prevented early pro-angiogenic cells from oxidative stress-induced apoptosis through downregulation of cleaved-caspase 3 . This result was in agreement with the results of the present study, which revealed that FOXO4 activation caused apoptosis in RCCs. Current research focuses on the tumor-suppressive roles of FOXOs and other target genes in the induction of apoptosis and their utilization for cancer therapy. FOXO3 was shown to bind to the promoter region of the Bim gene and enhance its transcription to promote apoptosis in breast (16) and hepatocellular carcinoma cancer cells (18), while downregulation of FOXO3 inhibits the transcription of its target gene Bim to decrease apoptosis (16). As members of the FOXO family, FOXO4 and FOXO3 show similar biological behaviors. Overexpression of FOXO4 was shown to activate the promoter of the Bim gene and increase its expression, resulting in an elevation of the apoptotic rate (16). Similarly, phosphorylation of FOXO4 was shown to trigger apoptosis, depending on the stimulation of pro-apoptotic protein Bim (19), which has a pivotal role in the control of mitochondria-dependent apoptosis. Consistent with these results, the present study also observed that FOXO4 caused apoptosis in cancer cells, partly through enhancing the expression of the Bim gene. Bim is a BH3-only protein, which can bind with all five pro-survival proteins (Bcl-2, Bcl-extra large, Bcl-w, A1 and myeloid cell leukemia 1), making it a more effective apoptosis inducer than other BH3-only proteins (20). However, the underlying mechanism of the interaction of $\mathrm{FOXO} 4$ with Bim requires further investigation.

In conclusion, the present study indicated that the knockdown of Bim in RCCs alleviated FOXO-induced mitochondria-dependent apoptosis. Although it is well known that FOXO4 exerts anti-tumor effects in numerous types of malignancy, the present study was the first to demonstrate its apoptotic effects in RCC. The present study enhanced the understanding of the complex underlying mechanisms of FOXO4-induced apoptosis, which was indicated to proceed via activation of the expression of the pro-apoptotic protein Bim, inducing mitochondria-mediated activation of the apoptotic cascade in RCC. Although it remains elusive how FOXO4 activates Bim expression, it represents a novel therapeutic target for RCC.

\section{References}

1. Chow WH and Devesa SS: Contemporary epidemiology of renal cell cancer. Cancer J 14: 288-301, 2008.

2. Moch H: An overview of renal cell cancer: Pathology and genetics. Semin Cancer Biol 23: 3-9, 2013.

3. Atkins MB, Choueiri TK, Cho D, Regan M and Signoretti S Treatment selection for patients with metastatic renal cell carcinoma. Cancer 115 (10 Suppl): S2327-S2333, 2009. 
4. van der Vos KE and Coffer PJ: The extending network of FOXO transcriptional target genes. Antioxid Redox Signal 14: 579-592, 2011.

5. Olmos Y, Sánchez-Gómez FJ, Wild B, García-Quintans N, Cabezudo S, Lamas S and Monsalve M: SirT1 regulation of antioxidant genes is dependent on the formation of a FoxO3a/PGC-1alpha complex. Antioxid Redox Signal 19: $1507-1521,2013$.

6. Brown J, Wang H, Suttles J, Graves DT and Martin M: Mammalian target of rapamycin complex 2 (mTORC2) negatively regulates Toll-like receptor 4-mediated inflammatory response via FoxO1. J Biol Chem 286: 44295-44305, 2011.

7. Alikhani M, Roy S and Graves DT: FOXO1 plays an essential role in apoptosis of retinal pericytes. Mol Vis 16: 408-415, 2010.

8. Cho EC, Kuo ML, Liu X, Yang L, Hsieh YC, Wang J, Cheng Y and Yen Y: Tumor suppressor FOXO3 regulates ribonucleotide reductase subunit RRM2B and impacts on survival of cancer patients. Oncotarget 15: 4834-4844, 2014.

9. Yu DA, Yoon J, Ko YS, Park J, Kim SY, Kim MA, Kim JH, Jung J, Cheon Y, Lee HS, et al: Forkhead transcription factor FOXO1 inhibits nuclear factor- $\kappa \mathrm{B}$ in gastric cancer. APMIS 122: 848-855, 2014.

10. Li H, Liang J, Castrillon DH, DePinho RA, Olson EN and Liu ZP: FoxO4 regulates tumor necrosis factor alpha-directed smooth muscle cell migration by activating matrix metalloproteinase 9 gene transcription. Mol Cell Biol 27: 2676-2686, 2007.

11. Lee MJ, Yu GR, Yoo HJ, Kim JH, Yoon BI, Choi YK and Kim DG: ANXA8 down-regulation by EGF-FOXO4 signaling is involved in cell scattering and tumor metastasis of cholangiocarcinoma. Gastroenterology 137: 1138-1150, 2009.

12. Su L, Liu X, Chai N, Lv L, Wang R, Li X, Nie Y, Shi Y and Fan D: The transcription factor FOXO4 is down-regulated and inhibits tumor proliferation and metastasis in gastric cancer. BMC cancer 14: 378, 2014.
13. Livak KJ and Schmittgen TD: Analysis of relative gene expression data using real-time quantitative PCR and the 2(-Delta Delta C(T)) method. Methods 25: 402-408, 2001.

14. Roy SK, Srivastava RK and Shankar S: Inhibition of PI3K/AKT and MAPK/ERK pathways causes activation of FOXO transcription factor, leading to cell cycle arrest and apoptosis in pancreatic cancer. J Mol Signal 5: 10, 2010.

15. Shukla S, Rizvi F, Raisuddin S and Kakkar P: FoxO proteins' nuclear retention and $\mathrm{BH} 3$-only protein Bim induction evokes mitochondrial dysfunction mediated apoptosis in berberine-treated HepG2 cells. Free Radic Biol Med 76: 185-199, 2014.

16. Lv Y, Song S, Zhang K, Gao $\mathrm{H}$ and Ma R: CHIP regulates AKT/FoxO/Bim signaling in MCF7 and MCF10A cells. PLoS One 8: e83312, 2013

17. Nakayoshi T, Sasaki K, Kajimoto H, Koiwaya H, Ohtsuka M, Ueno T, Chibana H, Itaya N, Sasaki M, Yokoyama S, et al: FOXO4-knockdown suppresses oxidative stress-induced apoptosis of early pro-angiogenic cells and augments their neovascularization capacities in ischemic limbs. PLoS One 9: e92626, 2014.

18. Carbajo-Pescador S, Steinmetz C, Kashyap A, Lorenz S, Mauriz JL, Heise M, Galle PR, González-Gallego J and Strand S: Melatonin induces transcriptional regulation of Bim by FoxO3a in HepG2 cells. Br J Cancer 108: 442-449, 2013.

19. Urbich C, Knau A, Fichtlscherer S, Walter DH, Brühl T, Potente M, Hofmann WK, de Vos S, Zeiher AM and Dimmeler S: FOXO-dependent expression of the proapoptotic protein Bim: Pivotal role for apoptosis signaling in endothelial progenitor cells. FASEB J 19: 974-976, 2005.

20. Dijkers PF, Medema RH, Lammers JW, Koenderman L and Coffer PJ: Expression of the pro-apoptotic Bcl-2 family member Bim is regulated by the forkhead transcription factor FKHR-L1. Curr Biol 10: 1201-1204, 2000. 